\title{
ESPACE PUBLIC, MÉDIAS, EFFETS ET STRATÉGIES DE RECONNAISSANCE
}

\author{
Jean-Louis Genard ${ }^{1}$
}

Les liens entre espace public, médias et reconnaissance ne sont nullement "naturels". Bien que structurels, ces liens prennent des formes très variables, déterminables à la fois par les diverses configurations historiques de l'espace public, et par l'évolution propre des modes de reconnaissance sociale.

En première approximation, on pourrait ainsi opposer, d'un côté une situation qui conjugue le faible niveau de problématicité de la question de l'identité sociale et le caractère avant tout politico-moral d'un espace public démocratique en voie de constitution -au XVIII ${ }^{\mathrm{e}}$ siècle-, et de l'autre -aujourd'hui- une situation où, au contraire, la question de la construction des identités est devenue socialement centrale et où l'espace public devient tendanciellement un espace des "relations publiques généralisées" 2 dans lequel la reconnaissance devient l'objet d'une quête autonome et organisée.

1 Professeur aux Facultés Universitaires Notre-Dame de la Paix (Namur), aux Facultés Universitaires Saint-Louis (Bruxelles), à l'Université Libre de Bruxelles et à l'École d'Architecture "La Cambre".

2 Cette expression est utilisée par B. MiEGE dans un texte intitulé "L'espace public: au-delà de la sphère politique", Hermès 17-18, Communication et politique, CNRS éd., Paris, 1995 , p. 49 et suivantes. 
Je chercherai donc, dans un premier temps, à distinguer les différents niveaux qui, historiquement, ont constitué l'espace public. Plutôt que de procéder, comme cela se fait souvent, à une appréhension globale de celui-ci, cette approche permettrait d'y déceler un certain nombre de strates obéissant à la fois à un principe de superposition, de contamination et de coexistence. A chacune de ces strates correspondent des effets, des formes et des stratégies de reconnaissance spécifiques, bénéficiant d'ailleurs de potentiels de légitimité variables. Ce sont ces processus que je dégagerai dans un second temps. Enfin, ces analyses invitent à saisir la compréhension des processus de reconnaissance médiatique en tenant compte à la fois de cette conception 'stratifiée' de l'espace public, mais également de la complexité des interférences qui peuvent s'instituer entre elles. C'est ce que je suggérerai pour terminer en évoquant l'idée d'une interprétation faisant droit à l'hybridité.

\section{Une conception "stratifiée" de l'espace public}

\section{L'espace public critique}

La première strate constitutive de l'espace public démocratique s'inscrit historiquement au cœur des crises qui, dès le $\mathrm{XVI}$ siècle, affectent les formes de la légitimité politique. Selon R. Koselleck ${ }^{1}$, la conception de l'État proposée par Hobbes accrédite tendanciellement l'idée selon laquelle les convictions, qu'elles soient religieuses, morales, esthétiques,... sont affaire privée alors que le rôle de l'État est essentiellement d'en organiser la coexistence. De ce processus, il va résulter, au niveau anthropologique, une division entre l'homme privé auquel est concédée l'aptitude à se forger de telles convictions et l'homme public, le citoyen, voué, chez Hobbes, à la seule obéissance. L'homme se divise donc entre le pôle de la passivité et de l'obéissance "citoyenne" et le pôle d'une conscience morale qui s'autonomise mais demeure alors vouée à l'inefficience politique.

Au-delà des limites inhérentes au modèle du Léviathan, on trouve là la marque d'une séparation entre État et individu et, surtout, le signe d'un renforcement et d'une autonomisation potentielles de l'instance individuelle par rapport à l'autorité étatique. A suivre

1 R. Koselleck, Le règne de la critique, Paris, 'Éd. de Minuit, 1979. 
Koselleck, cette autonomisation trouve sa justification théorique dans l'évocation de l'idée de "for intérieur". Cantonné d'abord à la seule sphère privée, celui-ci va connaître, avec le mouvement des Lumières, un processus de "dilatation"l qui en fera le point de référence dans la constitution de l'espace public démocratique. Koselleck insiste, à cet égard, sur la place occupée par J. Locke au travers de la distinction qu'il propose entre loi divine, loi de l'État et loi morale, qu'il appelle souvent "loi d'opinion et de réputation" 2 . Si, bien entendu, la loi de l'État conserve chez Locke une place prépondérante, en particulier en vertu de sa force coercitive, la loi morale en vient à être affectée d'un poids considérable, et cela d'ailleurs parce que, précisément, son autorité, avant tout morale, s'impose en l'absence de force coercitive.

La morale civile devient un pouvoir public qui n'agit que par l'esprit; elle est cependant politique, puisqu'elle oblige le citoyen à conformer ses actions non seulement aux lois de l'État mais aussi en particulier à la Loi de l'opinion publique ${ }^{3}$.

Même si, à l'époque, le caractère problématique de l'identité demeure marginal, on comprend déjà, au travers des expressions utilisées par Locke, en quoi l'espace public démocratique naissant portera ses effets sur l'image morale de ceux qui en sont les cibles. La loi d'opinion et de réputation est rapportée, par Locke, essentiellement à la question de la "vertu et du vice", et dès lors la question de la reconnaissance ("de l'estime et du blâme") n'est à ce moment en rien autonomisée mais se trouve rapportée à l'évaluation du contenu substantiel des actions. L'émergence de l'opinion publique n'est donc pas associée de manière directe à la question de la reconnaissance. Celle-ci est avant tout une autorité morale, susceptible de manière indirecte d'affecter la réputation de ceux qui en sont la cible.

Les représentants des positions morales n'ont sans doute pas de pouvoir politique, mais acquièrent en échange une force que son caractère exclusif rend écrasante ${ }^{4}$.

On peut penser que l'histoire de l'espace public est aussi celle de l'autonomisation de cette force "écrasante". Né de la disjonction du

I Ibid., p. 43.

2 Ibid., p. 44.

3 Ibid., p. 49.

4 lbid, p. 128. 
moral et du politique, l'espace public se construira désormais au cœur de leurs tensions.

La place prise par l'espace public en fait cependant très rapidement un lieu susceptible de produire des "effets de reconnaissance" en retour pour ceux qui y prennent position, incarnant en quelque sorte la conscience morale face aux agissements de pouvoirs qui, à l'époque, n'ont pas à en rendre compte. Et cela d'autant, je le répète, qu'il se constitue précisément en même temps que les anciennes formes "statutaires" d'identification sociale, attachées aux structures d'ancien régime, tendent à perdre leur autorité. On connaît ainsi l'extraordinaire notoriété dont ont pu bénéficier certains auteurs (Voltaire, Rousseau notamment) du fait de leurs prises de position au sein d'un espace public critique en train de se construire. Là se dessine un profil nouveau pour l'intellectuel qui annonce très certainement ce qui, plus tard, deviendra l'intellectuel médiatique. D'une certaine façon, ces premiers effets de reconnaissance seront liés à la nature même de cet espace public, sorte de conscience morale qui cherche sans y réussir pleinement à gagner une légitimité politique que les formes du pouvoir ne sont pas encore prêtes à lui concéder. Associées à la défense de convictions, les valeurs individualistes du courage, de l'honnêteté, ou de la sincérité y deviendront des instruments intrinsèques de valorisation.

Les armes de la critique seront les héritières de cette situation, mêlant conscience morale et impuissance politique. On y retrouvera les différentes figures du soupçon et de l'indignation morale, allant des figures de la dénonciation ou du tribunal, à celles plus féroces du pamphlet, ou encore à celles plus distanciées de la satire ou du cynisme.

\section{La question des minorités et de l'accès à l'espace public}

Cette conception de l'espace public centrée sur les idées de critique et d'engagement, qui prend essentiellement pour objet la sphère politique, qui numériquement demeure cantonnée à un public de propriétaires et de lettrés, et qui met en avant des intellectuels le plus souvent littéraires, va rapidement évoluer.

La première évolution significative est attestée par les prises de position de théoriciens libéraux qui, dans la première moitié du XIX siècle, en viennent à interroger l'espace public en termes d'accès 
(souvent en relation avec la question de la liberté d'expression) tout en associant cette interrogation à des appréciations ambivalentes quant à la valeur d'une opinion publique désormais évaluée en termes de conformisme potentiel et mise en cause quant à la pertinence, la rationalité et la justesse de ses jugements.

A cet égard, les analyses de J.S. Mill semblent exemplaires. Mill plaide en faveur de l'extension de la sphère publique, en particulier aux groupes qui en sont alors radicalement exclus. Il se montre particulièrement soucieux de garantir, par les libertés les plus larges, l'accès à la thématisation publique, et, au-delà à la visibilisation des questions politiques. A la même époque, politiquement, la question qui est au centre des débats, est celle de l'extension du suffrage. Une extension qui paraît structurellement inscrite dans les principes de la démocratie, mais qui se trouve très systématiquement thématisée au travers de l'opposition entre d'une part l'exigence de capacité critique et de rationalité (mesurée alors au caractère restrictif de l'espace public "bourgeois"), et de l'autre, le principe de la majorité ${ }^{1}$, exigences qui chez de nombreux auteurs de l'époque s'avèrent non seulement ne pas se recouvrir, mais plus résolument se contredire. Tout se passe donc comme si l'extension du suffrage s'accompagnait de l'émergence d'une suspicion accentuée à l'égard d'une "opinion publique" qui n'est plus seulement une conscience morale que l'on peut invoquer et porter à son crédit, mais bien aussi une puissance réelle susceptible d'agir politiquement (par le vote, les mouvements sociaux,...) et dont il convient désormais de pouvoir relativiser la force.

On assiste donc, à ce moment, à l'émergence de lieux à partir desquels une appréciation critique de l'opinion publique elle-même va devenir possible et légitime. L'opinion publique devient problématisable, ouvrant la voie aux diverses figures de la distanciation (avec ses thématiques de la manipulation, du conformisme, du $4^{\mathrm{e}}$ pouvoir,...).

C'est ainsi que les processus de démocratisation en viennent à générer des craintes spécifiques. Mill, par exemple, évoque un déplacement possible du lieu de la décision politique.

1 Voir à ce propos P. Rosanvallon, Le sacre du citoyen, Paris, Gallimard, 1992, p. 149-184. 
Le seul pouvoir digne de ce nom, écrit-il, est celui des masses, et celui des gouvernements en tant qu'ils se font les organes des tendances et des instincts des masses ${ }^{1}$.

Et Mill met clairement ce processus en relation avec l'émergence d'une opinion publique médiatisée par la presse :

la masse ne reçoit plus ses opinions des dignitaires de l'Église ou de l'Etat, de prétendus meneurs, ou des livres. Ses avis sont formés par des hommes très semblables à elle, qui s'adressent à elle ou parlent en son nom sous l'inspiration du moment, par l'intermédiaire des journaux ${ }^{2}$.

Sans que cela occupe une place centrale dans son argumentation, Mill introduit, conjointement à ces analyses, l'hypothèse d'un changement socio-anthropologique qui rappelle à bien des égards la thématique de la reconnaissance, telle qu'elle sera posée par D. Riesman un siècle plus tard. Plutôt que de s'auto-déterminer, l'individu démocratique en vient à adopter des comportements organisés en fonction d'une évaluation possible projetée sur autrui ${ }^{3}$. Or, poursuit Mill, la reconnaissance ne peut être le résultat d'une quête hétérodéterminée, mais seulement l'effet "secondaire" de la réalisation de soi $^{4}$. La crainte de Mill est celle d'une extension du conformisme et son plaidoyer pour la liberté s'avère dès lors aussi un plaidoyer pour la défense d'une possible reconnaissance des comportements minoritaires, des différences comme on dirait plus volontiers aujourd'hui.

Politiquement, émerge ici une deuxième strate de l'espace public à laquelle vont s'associer des formes spécifiques de reconnaissance, de valorisation ou de dévalorisation de l'opinion publique et des médias. Dénonciation du conformisme, de la "tyrannie de la majorité", et, inversement, souci des minorités et des différences, qui, en raison de leur statut minoritaire, risquent de se voir étouffées. Donner la parole à chacun dans un souci d's"objectivité" pour que le citoyen puisse "en conscience" juger et se déterminer. Mais surtout donner la parole à ceux dont les comportements sont en décalage par rapport à la majorité. On voit là se dessiner une nouvelle configuration des relations entre espace public, opinion publique et médias, configuration sans

I J. S. MiLl, De la liberté, Agora, n 67, Paris, Presses Pocket, 1990, p. 121.

2 Ibid., p. 121.

3 Ibid., p. 113-114.

4 Ibid., p. 117. 
doute liée à l'émergence du rôle et de la professionnalisation du journaliste, profession bénéficiant d'ailleurs à l'époque d'une image sociale largement péjorative. Là où précédemment l'intervention au sein de l'espace public supposait prises de position, engagement,... se dessine -en même temps que commence à s'autonomiser la sphère médiatique- l'image du retrait et de l'objectivité journalistiques attachée au souci de faire accéder au regard public des groupes, des pratiques, des comportements, des positions,... qui, sans cette intervention pourraient être vouées à l'anonymat, au refoulement social,... ou se trouver broyées par la suprématie de la majorité et du conformisme.

\section{L'émergence du "grand public"}

Comme je l'ai suggéré, l'évolution des structures de l'espace public au XIX $\mathrm{X}^{\mathrm{e}}$ siècle se manifeste notamment par un affaiblissement de l'exclusivisme de sa dimension politique. Affaiblissement intrinsèque lié à un libéralisme qui défend davantage un principe relativiste de tolérance que le principe critique qui avait prévalu auparavant. Mais affaiblissement extrinsèque également du fait de l'irruption au sein de l'espace public et des médias de préoccupations diverses tenant davantage de l'information générale ou du divertissement, qui, bien que traitées médiatiquement auparavant, l'étaient dans un contexte très spécialisé (les almanachs par exemple).

Alors que les craintes libérales se manifestaient essentiellement sur le terrain politique -et donc somme toute à l'intérieur de la logique initiale de l'espace public critique- l'émergence du "grand public" (et la multiplication des grands organes de presse qui l'accompagnera) va briser cette hégémonie du politique sur l'espace public.

Parallèlement à cette transformation, les frontières entre sphères privée et publique tendent à se fluidifier. Sans doute, ce processus n'est-il pas sans lien avec les diverses stratégies de censure qui émaillèrent le XIX ${ }^{\mathrm{e}}$ siècle, dissuadant de nombreux organes de presse de trop s'investir dans les affaires politiques. Mais il dénote également une transformation intrinsèque des rapports entre privé et public, corrélative à l'émergence de cette éthique de l'authenticité dont les effets s'accusent depuis la deuxième moitié du XVIII siècle. La presse -et singulièrement la presse à grand tirage, à vocation "populaire"- délaisse de plus en plus l'indignation politique pour lui 
préférer une indignation morale ambiguë où se mêlent un moralisme de bon ton et une hypocrisie qui permet de révéler le "vice" sous le couvert de la bonne conscience. Bien entendu, cette dénonciation peut prendre des formes généralisées, mais elle trouve surtout à s'extérioriser dans des formes particularisées, à tel point que pour de nombreux observateurs du XIX ${ }^{\mathrm{e}}$ siècle, une des fonctions essentielles de la presse y devient la construction et la destruction des réputations ${ }^{1}$. Ce tournant est attesté par les multiples conflits entre presse et justice autour de la diffamation qui marquèrent cette période. En particulier, la presse va entreprendre de concurrencer la justice et la police dans de multiples faits à "scandale". Les journalistes s'emploient à devancer les policiers dans la recherche des coupables et des disparus ${ }^{2}$, et n'hésitent pas à publier des documents couverts par le secret de l'instruction, se prévalant à chaque fois d'une référence à la liberté de la presse et à l'obligation morale du journaliste d'informer son public. L'autonomisation de la profession se marque notamment par la volonté des journalistes de s'instituer comme les garants des libertés ou du moins de certaines d'entre elles et de faire de critères internes -la déontologie ou la conscience- les critères exclusifs de l'exercice de la profession.

Éclatement et diversification des genres, règne du fait divers, interpénétration croissante du privé et du public, commercialisation massive du secteur, tels sont les éléments qui marquent en profondeur cette troisième strate constitutive de l'espace public démocratique.

\section{Le règne des "publicistes"}

G. de Tarde est sans doute un des premiers auteurs à conceptualiser les formes prises par l'espace public à l'ère de la communication de masse. Il entend clairement démarquer ses analyses des processus d'influence sociale des théories trop exclusivement centrées sur les foules, c'est-à-dire sur des rassemblements effectifs d'acteurs. Selon

\footnotetext{
1 On se rappellera les critiques virulentes, voire méprisantes, dont, à cette époque, les journalistes sont l'objet, notamment parce que leurs ambitions personnelles se réalisent souvent sur la destruction des réputations de ceux qui en sont la cible. On trouvera des illustrations de ce type d'argumentation dans les portraits de journalistes proposés par H. DE BALZAC dans les Illusions perdues.

2 On connait l'effet de ces interférences dans le roman policier, en particulier dans le personnage du journaliste détective illustré par Rouletabille.
} 
lui, l'âge des foules -selon l'expression avancée G. Le Bon- est progressivement en train de céder le pas à l'ère du public.

Dans cette perspective, Tarde en vient à très largement désubstantialiser les opinions. Loin d'être traitées en fonction de leurs prétentions critiques ou de leurs prétentions à la validité, comme c'était le cas des premières strates constitutives de l'espace public, les opinions sont envisagées sur le modèle de la consommation ou encore de la mode. Leur valeur de vérité cède très largement le pas aux effets d'adhésion qu'elles sont susceptibles d'engendrer. Nous sommes passés dans le registre de la foi, de la séduction -bref du "désir" pour utiliser la terminologie de l'auteur- et non plus dans celui de l'argumentation ou de la justification. Il faut bien entendu replacer cette approche dans le contexte théorique plus global qui situe Tarde parmi ces sociologues qui, suite aux travaux des spécialistes de la psychopathologie ${ }^{1}$, avaient trouvé dans l'hypnose ou le somnambulisme le modèle de l'interaction sociale. L'opinion est, à cet égard, par excellence un vecteur d'imitation ou de contagion mentale.

Tarde associe cette transformation de l'espace public à l'irruption d'une forme nouvelle d'acteur social, irruption favorisée par un certain nombre de phénomènes sociaux contextuels, développement des villes, déstructuration des relations sociales primaires... Un acteur social dont, précisément, l'identité se trouve moins assurée et qui, dès lors, devient éminemment tributaire de ce que Tarde identifie en parlant du regard d'autrui ${ }^{2}$. Ce contexte, insécurisant les identités, vouant les interactions sociales à s'organiser sous "la pensée du regard d'autrui", favorise l'apparition sur la scène publique et médiatique d'un nouveau type d'acteur qu'il qualifie de "publiciste", personnage dont la trajectoire ne peut se comprendre que dans ses interactions avec le public. Ce que Tarde appelle "publiciste" est en fait un personnage qui concentre en lui ou plutôt sur lequel se concentre, se focalise un courant d'opinion. Le publiciste est le porteur, l'interprète d'un mouvement social, au départ peut-être latent, mais auquel il va donner forme et consistance. Sans de tels publicistes, les mouvements sociaux seraient voués à demeurer à l'état latent ou léthargique. Le rapport du publiciste à son public mêle à la fois fascination et consommation ${ }^{3}$. Il n'a plus que très peu de choses à voir avec le

\footnotetext{
1 Voir J.-L. Genard, Sociologie de l'éthique, Paris, L'Harmattan, 1992, p. 21-32.

2 G. DE TARDE, L'opinion et la foule, Paris, PUF, 1989, p. 34.

3 Tarde parle, à ce propos, de "clientèle commerciale" (Ibid., p. 42).
} 
contenu de vérité des positions qu'il représente. Parmi les publicistes, Tarde range à la fois des leaders de mouvements sociaux -politiques ou non-mais aussi les vedettes internes au monde médiatique, en particulier les journalistes.

[Les publicistes], bien plus que les hommes d'État, même supérieurs, font l'opinion et mènent le monde. $\mathrm{Et}$, quand ils se sont imposés, quel trône solide est le leur! Comparez à l'usure si rapide des hommes politiques, même des plus populaires, le règne prolongé et indestructible des journalistes de haute marque, qui rappeile la longévité d'un Louis XIV ou le succès indéfini des comédiens et des tragédiens illustres. Il n'est pas de vieillesse pour ces autocrates ${ }^{1}$.

Selon Tarde, ce processus est appelé à transformer radicalement les formes de la communication et de l'espace publics. De l'espace, parce que la reconnaissance de tout mouvement social passera désormais par sa médiatisation ${ }^{2}$ et par son aptitude à assurer l'émergence d'un publiciste qui le fédérera et qui, au travers de sa reconnaissance personnelle en assurera la reconnaissance sociale. De la communication publique, parce qu'avec l'émergence des publics, les processus d'adhésion se feront bien davantage sur des bases affectuelles, ce qui infléchira forcément les modes d'interaction avec le public, et cela au profit de stratégies excessives, adoration ou apologétique d'un côté, haine et diffamation de l'autre. Tarde voit d'ailleurs dans ce processus un élément essentiel de transformation des structures publiques démocratiques, en train de passer à la démocratie de masse. Pour lui, il ne fait pas de doute que les évolutions récentes de la démocratie sont très clairement attachées à la fois, bien sûr, au suffrage universel, mais aussi et peut-être surtout au journalisme ${ }^{3}$. Et il se montre d'ailleurs très circonspect à l'égard de l'avenir des démocraties: "le danger des démocraties nouvelles, écrit-il, c'est la difficulté croissante pour les hommes de pensée d'échapper à l'obsession de l'agitation fascinatrice" 4 .

La dichotomisation entre la pensée critique autonome et le règne de l'opinion qui, comme on l'a vu, s'était lentement creusée, est à ce

\footnotetext{
1 Ibid., p. 44.

2 Tarde écrit par exemple: "un parti sans journal ne nous fait-il pas l'effet d'un monstre acéphale?" (Ibid., p. 45.).

3 Ibid., p. 73.

4 Ibid., p. 71.
} 
moment devenue béante. A suivre Tarde, l'espace public démocratique pourrait très bien s'orienter de manière telle que l'indépendance de l'intellectuel critique cède la place à la séduction de la vedette médiatique.

\section{Le règne des "relations publiques généralisées"}

La strate précédente, décrite en référence aux travaux de G. de Tarde, insistait essentiellement sur un certain nombre d'effets résultant de l'importance prise progressivement par le développement des médias de masse et par les transformations des processus d'influence qui en résultaient. Le développement des relations publiques généralisées correspond à un approfondissement de cette logique, mais un approfondissement qui va en modifier la nature. Nous nous trouvons cette fois -à une période dont il conviendrait sans doute de situer l'émergence aux alentours des années 1950-1960- face à un système médiatique qui s'est largement autonomisé et qui tend par une sorte d'effet d'entraînement à propager, à disséminer sa logique dans l'ensemble des processus sociaux où se posent des questions d'influence, de communication ou d'image. Cette logique fait l'objet d'investigations autonomisées, de processus de spécialisation (marketing, services spécialisés dans les entreprises...). Se développent donc des stratégies volontaristes orientées vers la création d'images de marques, vers l'entretien et le développement de réseaux relationnels... Loin d'être l'effet de processus extérieurs à sa recherche, la reconnaissance devient dès lors l'objet d'une quête autonome, organisée, résultant de stratégies minutieusement préparées. Cela sans doute à partir de l'autonomisation et de la spécialisation du secteur publicitaire (les premières grandes agences publicitaires internationales naissent aux alentours de la seconde guerre mondiale). Dans certains secteurs d'activités, ce type de processus deviendra dominant, voire exclusif (pensons au show-business), produisant des processus de reconnaissance d'une nature particulière, jusque là peu imaginables, entrée fulgurante dans le vedettariat ou passage du vedettariat médiatique à la sphère politique, par exemple.

Le système politique lui-même s'en trouve très rapidement affecté même si, au départ, ces logiques pénétreront d'abord d'autres sphères sociales, en particulier la sphère économique. Transposition de la logique du marketing économique vers la sphère politique. 
Formation des hommes politiques à l'utilisation des médias, "petites phrases", pratique généralisée de la conférence de presse, désignation de porte-parole... De manière plus générale, les médias vont devenir un lieu privilégié d'enjeux et d'investissements de la part de la classe politique, et cela vraisemblablement aux dépends des lieux de l'action politique qui prévalaient auparavant (militantisme de base, action dans la rue...). Les débats publics s'en trouvent potentiellement davantage encore désubstantialisés. Mais surtout ils se trouvent dédoublés ou court-circuités par des processus -quelquefois totalement occultésde relations publiques. Le règne des relations publiques généralisées est, en effet, aussi, au niveau de l'influence politique, celui qui met en avant la pratique généralisée d'un lobbying qui est souvent le complément occulte des stratégies plus apparentes d'image de marque.

Pris dans le jeu, les médias deviennent à la fois les juges et les arbitres, mais aussi les instruments de stratégies d'influence qui, le cas échéant, les dépassent.

\section{Les effets de système et l'explosion d'une communication auto-référentielle}

La dernière strate que je souhaiterais évoquer est celle qui résulte de manière directe de l'autonomisation croissante du sous-système médiatique et des effets "auto-poiétiques" (selon l'expression de $\mathrm{N}$. Luhmann) qui en résultent.

Je vise par là le développement et la multiplication de processus médiatiques justifiés par les médias eux-mêmes. Processus auto-référentiels qui supposent une sorte de bouclage sur elle-même de la communication médiatique, et dont la fonction est, cette fois de manière directe, l'auto-alimentation, l'auto-célébration ou l'autoreconnaissance. Présentateurs invitant les journalistes, journalistes invitant les présentateurs, humoristes imitant les présentateurs, journalistes célébrant les journalistes... Les exemples pourraient être multipliés. A ce moment les critères d'évaluation des médias tendent à devenir eux-mêmes autonomes et auto-référentiels. C'est la toute puissance de l'auto-évaluation illustrée par l'audimat. Logique qui d'ailleurs affecte les sphères qui vivent sous la dépendance -au moins partielle- des médias: baromètre de popularité des hommes politiques, hit parade des images d'entreprises. 
Cette logique auto-référentielle contamine d'ailleurs les autres sous-systèmes sociaux obligés de se prêter au jeu sous peine d'invisibilité sociale. C'est ainsi que l'on voit des hommes politiques s'abandonner à la logique médiatique, participant à des jeux télévisés, se prêtant à des interviews dans l'intimité... Le passage, la "pénétration" médiatiques deviennent des critères intrinsèques de valeur.

Les différents médias, couplés aux secteurs qui en sont les sources d'alimentation privilégiées, en viennent progressivement à former entre eux un système auto-suffisant, et cela d'autant que se renforcent des empires médiatiques pluri-médias. Télévision, presse écrite, monde des variétés... parlent des mêmes choses, mettent en scène les mêmes personnages, qui ne sont plus que, d'une certaine façon, des effets médiatiques.

\section{Les strates de la reconnaissance}

Cette approche historique a permis de cerner brièvement les différents niveaux constitutifs de l'espace public démocratique. Comme je le signalais d'emblée, ces niveaux obéissent à la fois à un principe de superposition, les niveaux supérieurs tendant à supplanter ceux qui les précèdent historiquement, mais aussi à un principe de stratification, les niveaux inférieurs continuant de produire leurs effets propres, tout en voyant bien entendu ces effets infléchis par les niveaux supérieurs.

Fort de cet acquis, je souhaiterais aborder maintenant de manière plus approfondie la question de la reconnaissance. A chacune de ces strates correspondent en effet des formes, des effets et des stratégies de reconnaissance spécifiques, qui ne sont d'ailleurs pas sans lien avec l'émergence et le déploiement, au niveau social global, de la thématique de la reconnaissance et des éthiques de l'authenticité, telles qu'elles sont théorisées par $\mathrm{Ch}$. Taylor ${ }^{1}$.

Il s'agira donc, dans cette deuxième partie du texte, et dans une optique proche de l'articulation proposée par L. Boltanski et L. Thévenot ${ }^{2}$ entre "cités" et "mondes", de faire correspondre à chacune des six strates constitutives de l'espace public contemporain, six "mondes" de la reconnaissance (ce qui y accède factuellement à la

1 Ch. TAYlor, Le malaise de la modernité, Paris, Éd. du Cerf, "Humanités", 1994.

2 L. Boltanski et L. ThÉvenot, De la justification, Paris, Gallimard, coll. Essais, 1991. 
reconnaissance, ce qui y est considéré comme normativement digne d'accès à la reconnaissance...) en les illustrant par des processus médiatiques actuels. Les exemples cités seront cette fois rigoureusement contemporains. Ils seront choisis parce qu'ils illustrent particulièrement telle ou telle strate. Toutefois, imbriqués au sein d'un espace public et médiatique stratifié, ils seront bien souvent susceptibles d'interprétations plus nuancées que j'aborderai dans la partie terminale de ce texte.

\section{Le courage de la critique}

Cette première forme de reconnaissance correspond aux strates les plus profondes de l'espace public, en particulier celle héritée du XVIII' siècle, bien qu'elle puisse, je le répète, se trouver infléchie par les strates plus récentes.

Est susceptible d'être reconnu à ce niveau celui qui est capable, qui ose critiquer, dénoncer les dysfonctionnements, les injustices, les malversations... propres aux sphères du pouvoir, en particulier du pouvoir politique; comme d'ailleurs les excès, les abus, les corruptions de ceux qui le détiennent. Il est probable que la reconnaissance sera d'autant plus "pure", d'autant plus légitime, que les dénonciations viseront -au-delà des critiques personnalisées qui, souvent, font le prix du courage de la dénonciation- des problèmes impersonnels, écarts aux exigences institutionnelles, manquements objectifs... bien davantage que les personnes en tant que telles. Même si d'autres strates de l'espace public appellent cette personnification de la dénonciation. Il y a d'ailleurs là toute une dialectique, une rhétorique qui s'instaure entre dénonciations personnalisées et objectivantes, où le risque d'être taxé de poujadisme, de jalousie... le dispute souvent à l'exigence de "prendre ses responsabilités", d'apporter des preuves et de dire des noms.

Le crédit de celui qui dénonce sera d'autant plus vaste qu'il n'est pas personnellement intéressé, que les risques qu'il prend sont grands et, surtout, que ses chances de s'affirmer, d'arriver à résister ou tout simplement d'être entendu... étaient, au départ du moins, minces. Soit que la puissance de l'appareil de pouvoir paraissait disproportionnée par rapport à celui qui contestait, soit que la cause initiale semblait a priori perdue. La disproportion, la dissymétrie des positions est ici un facteur essentiel de valorisation, dissymétrie en nombre (l'homme seul 
face au pouvoir, face au système...), dissymétrie en force sociale (l'absence de moyens, de ressources...). Ce que celui qui dénonce a pour lui, c'est la certitude et le courage de celui qui, contre vents et marées, défend une cause juste ou son honneur et qui, pour cela, est prêt à prendre les plus grands risques, le cas échéant le risque de sa vie. L'opinion publique est pour lui la ressource à laquelle il en appelle. Les médias jouent pour lui le rôle d'intercesseur, représentant parfois l'espoir ultime. Ils sont l'instance qui permettra à la cause de se faire entendre, à la justice d'obtenir droit de cité, qui donnera à l'honneur bafoué une ébauche de réhabilitation, qui forcera ceux qui sont injustes à surseoir aux pressions, à entrer en dialogue. C'est ce qui explique ce surinvestissement pathétique dont sont parfois l'objet les médias de la part de ceux qui crient désespérément à l'injustice.

Les médias occupent ici une position potentiellement engagée. En prenant fait et cause pour celui qui s'indignait sans se faire entendre, en dévoilant au grand jour ce que l'on cherchait à dissimuler... les médias bouleversent les donnes de l'espace public et reçoivent des effets de valorisation en retour. Leur mode d'intervention peut être celui, moral, de l'indignation (dans un rapport de plus ou moins grande proximité avec ceux à qui est donnée la parole); ou celui, plus objectivant, de l'analyse minutieuse et documentée; mais il peut être celui plus juridique du procès ou du tribunal (formes d'ailleurs héritées de l'espace critique du XVIII' siècle). Les médias entretiendront à cet égard avec la sphère juridique des rapports étroits, où se mêlent connivences et conflits, relayant certains processus internes au champ juridique, donnant la parole aux juges "intègres", aux "petits" juges, aux causes qui risquent de ne pas être entendues, dénonçant les pressions que subissent juges et magistrats... On pensera ici au rôle des médias dans la visibilisation de l'affaire du sang contaminé, ou encore au soutien médiatique des juges d'instructions s'occupant de corruptions...

Ceux qui sont ainsi capables de s'indigner pour des causes, de s'opposer à des pouvoirs... en viennent à former "couple" avec ceux qui -dans les médias ou dans ses alentours- lui apportent soutien, caution... Nous penserons ici aux relations des médias avec des personnages comme les dissidents des pays de l'Est, S. Rushdie, T. Nasreen... Mais également au statut des divers comités de soutien, pétitions... qui se créent à cette occasion; au statut des journalistes placés dans des contextes de crise, par exemple aujourd'hui en Algérie (statut qui fait bien entendu beaucoup pour le crédit moral global de la 
profession), au statut de certains intellectuels médiatiques (B. Kouchner, B.H. Lévy, A. Glucksman...) qui font profession d'indignation, ou encore aux effets de reconnaissance en retour dont peuvent se prévaloir les personnages médiatiques qui cautionnent ou soutiennent ceux qui risquent leur vie pour une cause (pensons à certaines invitations de B. Pivot, souvent sous la forme sacralisée d'émissions spéciales)...

\section{La sollicitude à l'altérité}

A partir d'une position qui est cette fois davantage objectivante (plutôt faire voir que partager une indignation), il s'agit ici de faire accéder à la visibilité et donc à certaines formes de reconnaissance ceux qui sont minoritaires, exclus, laissés pour compte... Ceux qui sont ainsi l'objet de ces processus se trouvent effectivement mis au centre de mécanismes de reconnaissance, que celle-ci soit individuelle ou de groupe. L'attention qui leur est ainsi portée -parce qu'elle a moins pour objet la cause qu'ils représentent que les personnes qu'ils sont- brisant bien souvent les frontières entre le public et le privé, fait appel à des modes d'adhésion plus chargés affectivement (sollicitude, compassion, sympathie...). La reconnaissance passe en effet ici bien davantage par un processus d'exposition. De manière très générale, cette forme de visibilisation de l'altérité et de la différence trouve dans le développement actuel des éthiques de l'authenticité l'occasion de son épanouissement, au travers d'un répondant massif des téléspectateurs à de telles sollicitations médiatiques (le succès des émissions de M. Dumas en offre une bonne illustration).

Cette reconnaissance n'est évidemment pas sans lien avec la reconnaissance en retour qui peut rejaillir sur ceux qui font accéder à la visibilité ceux qui ont été oubliés, refoulés, ceux dont on ne parle pas. De sorte que s'instaurent ici également des relations complexes entre acteurs qui, quelquefois, appartiennent à des mondes totalement différents. Pensons aux passages médiatiques du chanteur Sting accompagné d'un chef indien pour plaider la cause des populations autochtones et de la forêt d'Amazonie. Les motivations de l'accès à la visibilité peuvent évidemment être très variables, allant des dimensions les plus politiques, proches de la strate précédente (les grands reportages sur les exclus...), aux dimensions centrées sur le recherche pure de l'insolite, de la différence pour la différence qui nous amènent 
vers des strates orientées essentiellement vers l'auto-reconnaissance médiatique (pensons aux émissions animées par A. de Caunes et J.P. Gaultier sur MCM), en passant par les dimensions plus objectivantes (dans le chef du présentateur) propres aux strates intermédiaires (pensons ici à l'émission Bas les masques de M. Dumas).

\section{La célébration du fait divers, de la banalité et de l'anodin}

Avec le traitement du fait divers, nous assistons à l'entrée, le plus souvent (mais pas toujours) éphémère, de personnages anonymes sur la scène publique. Les cloisons entre ce qui est digne d'intérêt et ce qui ne l'est, comme entre ceux qui sont dignes d'intérêt et ceux qui ne le sont pas, s'estompent. C'est l'émergence de la "masse", comme spectateur d'abord, comme acteur ensuite. Aux effets de reconnaissance passagers de ceux qui sont mis sous les feux de la rampe, répondent les effets de reconnaissance de ceux qui mettent cela en scène, dans des formes plus ou moins orientées vers le vedettariat médiatique et avec des motivations explicites également variables. Intérêt quasi ethnologique (Faits divers de la RTBF), motivations policières (Témoin $n^{\circ} \mathrm{l}$, Perdus de vue...)...

A côté de ces productions qui demeurent motivées par le fait divers -avec ses connotations potentiellement dramatisables-il faut également insister sur celles qui tendent à une désubstantialisation radicale de leur contenu. S'appuyant ou bien sur la mise en scène de l'inattendu, de l'exceptionnel... ou au contraire du banal, de l'anodin, de l'anonyme... elles sont l'occasion d'organiser le passage médiatique d'acteurs, de faits qui, sans cela, n'auraient pu y prétendre, ou encore d'acteurs qui, connus médiatiquement, n'auraient pu être, pour cela, l'objet d'un traitement médiatique. D'un côté, des émissions qui, comme celle animée par J.L. Morandini sur TF1 (Tout est possible...) insistent sur l'exceptionnel, l'inhabituel...; de l'autre, par exemple, les jeux télévisés, et spécialement parmi ceux-ci ceux qui ne font appel à aucune "culture" particulière (La roue de la fortune, Une famille on or, la brosse à dents de Nagui...), qui ne sont plus que la mise en scène médiatique de téléspectateurs anonymes. Rendues possibles par l'autonomisation et l'auto-référentialité médiatiques, trouvant prétexte dans le jeu, la valeur des prix, souvent avec la référence centrale à la chance (on connaît le succès des jeux dans les populations occidentales, de même d'ailleurs que le rôle de la chance dans la construction 
d'un rapport fantasmatique à sa propre trajectoire sociale), se construisant sur la célébration de l'anodin et du quotidien, ces émissions sont l'occasion de la mise en scène d'une personne, d'une famille, héros éphémère d'un passage médiatique, créé par les médias eux-mêmes. Ces productions trouvent leur complément dans l'image de présentateurs, affables, enjoués, complaisants, "sympathiques"... qui fondent une part de leur popularité dans des mises en scène souvent orientées par l'affaiblissement des distances téléspectateurs-médias (émissions "populaires", "sans prétention"...).

\section{Le personnage médiatique}

Avec celui-ci (dont le prototype pourrait être B. Tapie), nous entrons de plein pied dans une logique où, les opinions se trouvant très largement désubstantialisées, la question de la valeur de la personne est mesurée à sa capacité de construire et de rassembler un public. Le rapport du public à la vedette médiatique relève essentiellement, comme le suggère Tarde, de la fascination et de la consommation. S'instaurent alors des relations complexes entre le personnage et son ou ses publics, relations articulées avant tout par le façonnement et la construction par et au travers des médias d'une "image". Relations dans lesquelles les journalistes ou présentateurs en viennent quelquefois à prendre le statut de faire-valoir. L'apparition médiatique du personnage relèvera du "show", soigneusement préparé et mis en scène. Il s'autorisera éventuellement des libertés par rapport à la "bienséance" médiatique. Il remettra le présentateur à sa place, agissant au nom du courant ou du public qu'il est censé représenter, ou encore au nom des responsabilités "qu'il assume, lui". Il n'hésitera pas à critiquer les médias, à revendiquer pour lui une majorité silencieuse... mettant en question les opinions "soi-disant majoritaires". Contre celui supposé des médias, il pourra proposer son propre "agenda".

Les critères de la valeur médiatique du personnage se sont désormais déconnectés des mécanismes d'adhésion à ses positions ou à ses discours. L'audience potentielle devient un critère intrinsèque d'évaluation. Ce fut, en France, le cas de G. Marchais dont les apparitions télévisées suscitaient un intérêt d'audience sans commune mesure avec la représentativité politique du parti communiste. 
L'image médiatique du personnage est attachée à une logique multiforme de présence où se brisent les barrières entre médias (il sera exploité peut-être avec distance par le Monde, mais aussi par la presse à sensations), entre genres médiatiques (il sera aussi bien l'invité d'Anne Sinclair, que de J.-P. Foucault; il aura sa place aux Guignols de l'Info, sera piégé par une caméra cachée...), de même, bien entendu, que celles qui opposent privé et public. L'entretien de cette image -qui, pour les médias, fonctionne comme un capital- se doit d'être entretenue. Entretenue par le personnage qui gagne à cultiver ses différences, ses excès...-bref ce qui l'identifie et le distingue-; mais entretenue également par les médias eux-mêmes qui gagnent à multiplier les occasions où le personnage donnera sa "pleine mesure". On pensera ici aux stratégies mises en place par les médias autour de B. Tapie ou encore de J.M. Le Pen.

Cette logique ne manque évidemment pas d'infléchir les comportements médiatiques des hommes publics qui eux aussi cherchent à détenir ou à entretenir une "image", à assurer leur visibilisation. Soit par des stratégies directes de construction d'image, soit en cherchant à se situer par rapport aux personnages médiatiques (en les critiquant durement, en les ignorant ostensiblement, en cherchant à partager avec eux des présences médiatiques...).

\section{Porte-parole, sponsoring, marketing}

Avec le règne des relations publiques généralisées, se construit une logique où est clairement et explicitement prise en compte comme finalité de l'action l'exigence de reconnaissance médiatique. Entre médias et relations publiques s'instaurent des liens d'instrumentalisation réciproque. D'une logique d'effets de reconnaissance, nous sommes maintenant très clairement passés à une logique volontariste. Les stratégies des acteurs (individuels, mais souvent collectifs, groupes de pression, entreprises, partis...) s'en trouvent considérablement infléchies. Individualisation et personnalisation de l'action (qui posera question à des mouvements sociaux dont les idéaux s'en accommodent mal); investissement dans des activités "rentables" médiatiquement (sponsoring...), transformation des formes du discours public (porte-parole, conférence de presse...), valeur des personnes évaluée à leur pénétration médiatique (sondages...), mise en scène et célébration de l'image (anniversaires, cocktails, vernis- 
sages...) Se créent alors des réseaux relationnels, de dépendance et de connivence mutuelles entre monde médiatique et monde des relations publiques.

Le monde médiatique en vient à vivre en symbiose totale avec un certain nombre de mondes connexes -variété, cinéma, mode...- mais impose également sa logique à des mondes plus autonomisés (économique, politique, recherche...). Les premiers, qui vivent essentiellement d'une logique d'opinion, trouvent dans les médias leur support indispensable. Ainsi, le monde du show business a-t-il "squatterisé" les émissions de variétés (logique promotionnelle, hit parade, clips....), pour en arriver à générer des chaînes télévisées spécialisées (MTV...), assurant une autonomisation radicale des processus de reconnaissance interne au champ. De même, le monde du cinéma en est arrivé, en l'espace de quelques années, à modifier radicalement la nature des émissions télévisées qui y sont consacrées, faisant de ces émissions de purs instruments promotionnels organisés autour de la diffusion de bandes annonces proposées par l'industrie cinématographique elle-même. L'activité de promotion fait désormais partie intégrante de toute activité de production, mobilisant ses vedettes lors de la commercialisation des films, multipliant les passages médiatiques, ajustant les dates de sortie sur des événements médiatisés (festivals...), accompagnant les grandes productions de stratégies de "merchandising" savamment calculées... Les "causes" médiatiques (le Sida, l'humanitaire...) deviennent des vecteurs de promotion, comme dans le cas de ces grands couturiers qui organisent leurs défilés à l'Unesco, ou encore au profit d'entreprises caritatives, ou encore dans le cas des publicités de Toscani pour la firme Benetton.

Les secteurs plus autonomisés ne sont pas en reste. Certains, comme le sport -ou du moins un certain nombre d'entre eux (Formule 1, football, cyclisme, tennis...)- se trouvent totalement annexés par la logique des relations publiques généralisées, à tel point que les vedettes sportives deviennent de véritables personnages médiatiques, dont la pénétration médiatique transgresse très largement les frontières de la discipline (pensons aux cas de E. Cantona, d'A. Senna...). D'autres, comme la politique ou l'entreprise, entretiennent des rapports étroits, quasi symbiotique, avec les médias, "médiatisation" des PDG (A. Aflelou,...), politique de présence dans les occasions médiatiques, association - via le sponsoring-à des mani- 
festations culturelles, sportives ou caritatives, adhésion ostensible à des exigences éthiques...

Parallèlement à ce processus, on assiste à une autonomisation de la logique des relations "publiques" elle-même. Celles-ci tendent alors à prendre leurs distances par rapport au principe de publicité inhérent à la logique médiatique. C'est la pratique -souvent occulte- du lobbying qui renvoie les processus d'influence vers la pratique du secret.

\section{Auto-célébration et auto-référentialité}

Si les strates précédentes laissaient déjà apparaître ce que l'on pourrait appeler des effets de champ ou de système, on assiste maintenant à une sorte d'aboutissement de ce processus avec l'émergence d'effets de bouclage, d'auto-alimentation, d'auto-célébration... La communication devient très largement auto-référentielle, elle ne produit plus que l'auto-reconnaissance du champ par lui-même. Invitations mutuelles de présentateurs, d'animateurs (l'émission récemment disparue, qui était animée par $M$. Denisot sur Canal+ le dimanche en début de soirée...), culte de l'audimat, du scoop ou de l'exclusivité, célébration de l'actualité ou de l'histoire du média (Plateaux télé, les "enfants de la télé" animée par Arthur et P. Tchernia, sur A2, les bêtisiers, les rétrospectives...), prix attribués aux professionnels, présence ostensible du monde médiatique aux cérémonies médiatisées...

\section{L'hybridité comme vecteur d'interprétation}

Il va sans dire que ces "mondes de la reconnaissance" doivent être pris comme des types idéaux et que, dès lors, l'analyse de productions médiatiques spécifiques nécessite le plus souvent, comme cela a d'ailleurs déjà été souligné, la prise en compte simultanée de strates différentes qui entretiennent des rapports complexes, se renforçant mutuellement, euphémisant ou cherchant à annuler certaines d'entre elles...

Cette hybridité est très certainement une source de confusion dans les interprétations des productions médiatiques contemporaines. Et cela parce que, très souvent, ces analyses ne font pas droit à la complexité stratifiée de l'espace public, lui préférant une conception 
plus monolithique, s'interdisant dès lors de prendre au sérieux des prétentions pourtant revendiquées par telle ou telle production médiatique.

Je souhaiterais terminer cet article en évoquant certaines pistes d'analyse basées précisément sur la mise en évidence de l'hybridité des processus et effets de reconnaissance qui y sont sous-jacents.

Je commencerai par l'exemple des Guignols de l'Info. Cette émission me semble particulièrement intéressante parce qu'elle s'appuie sur l'association des strates extrêmes qui constituent l'espace public contemporain autorisant à la fois toutes les critiques et toutes les dénégations. On trouve en effet, au coeur de la logique de cette émission, toute une série de caractéristiques qui, à l'évidence, la tirent vers les strates les plus récentes de l'espace public. Mise en scène du journalisme par lui-même, écran dans l'écran, auto-dérision, imbrication du politique et de la variété, réduplication de la réalité médiatique... Mais en même temps l'objet et le ton de l'émission la rapportent plutôt aux strates plus profondes de l'espace public. Thématiques politiques, virulence à l'égard de la classe politique, critique des médias... L'évaluation d'une telle émission s'avère dès lors complexe parce que sa structure autorise toutes les dénégations. Ainsi, par exemple, l'accusation de facilité trouvera-t-elle à s'euphémiser dans l'attestation d'une indépendance d'esprit et d'un ton sans complaisance à l'égard de la classe politique.

Dans un registre très semblable, on pensera au cérémonial propre au Festival de Cannes qui, en tant que tel, renvoie très largement aux niveaux 5 et, surtout, 6 dégagés précédemment, suscitant dès lors les critiques attendues qui y correspondent (caractère commercial, snobisme...). Dans ce contexte, le fait même d'y donner un prix important à un acteur trisomique, tend à briser ces seules dimensions et, du coup, fait rejaillir sur la manifestation des effets de reconnaissance liés à d'autres strates (en particulier la strate 2). Ce qui va autoriser une pénétration, par le biais mais dans un registre sérieux, d'un festival volontiers critiqué dans des émissions à vocation informative et critique (La marche de siècle). De la même façon, des manifestations comme le Sidaton, le Téléton, ne sont interprétables qu'au travers de l'imbrication entre des effets de champ (strates 4,5 et 6) et des effets de reconnaissance propres aux strates plus profondes de l'espace public. Cette ambivalence ne manque d'ailleurs pas de susciter des tensions entre les acteurs de "terrain" qui reprochent à l'initiative son 
côté spectaculaire et les professionnels des médias forcés alors de produire une auto-justification de la forme spectaculaire utilisée

Enfin, mais en sens inverse cette fois, les stratégies de revendications politiques ne sont plus aujourd'hui compréhensibles sans une analyse de leurs rapports avec les strates les plus récentes et superficielles de l'espace public. Pensons à la tentation de certaines organisations (par exemple Amnesty International) de produire des sortes de "hit parade" des atteintes aux droits de l'homme. Ou encore au devenir spectacle de la revendication politique, telle qu'elle peut être illustrée par Greenpeace. 